\title{
Changes in psychiatric nurse attitudes towards legal representation of inpatients at district psychiatric board hearings in Israel: a pilot study
}

\author{
Alexander Grinshpoon ${ }^{1}$, Razek Khawaled ${ }^{2}$, Tamar Levy ${ }^{1}$, Paula Rosca ${ }^{2}$, Alexander M. Ponizovsky ${ }^{2 *}$ \\ ${ }^{1}$ Tirat Carmel Mental Health Center, Tirat Carmel, Israel; \\ ${ }^{2}$ Mental Health Services, Ministry of Health, Jerusalem, Israel. \\ Email: ${ }^{2}$ alexander.ponizovsky@moh.health.gov.il
}

Received 11 August 2011; revised 8 September 2011; accepted 16 September 2011.

\begin{abstract}
Aims: To compare the initial attitudes of nurses and other professional groups in a psychiatric hospital towards the legal representation (LR) of involuntary admitted psychiatric patients before District Psychiatric Boards (DPB), and to assess how these attitudes changed after LR had become an everyday routine practice in the hospital. Background: LR of hospitalized psychiatric patients before DPB has been introduced into mental health system worldwide, includeing Israel. Attitudes of psychiatric staff towards LR and their changes over time are a very important -though unstudied issue. Methods: Thirty seven psychiatric nurses employed in the Tirat Carmel Mental Health Center, Israel and 30 other mental health professionals (comparison group) completed the Attitudes to Legal Representation Questionnaire before LR has been instituted in the Center (November 1, 2006) and one year later. Baseline differences between the groups were tested using one-way ANOVA, and pre-post differences in the attitude scores were tested by paired t-tests. Results: Baseline psychiatric nurses attitudes towards LR were significantly more negative than those of the comparison group $\left[F_{67,3}=\right.$ $6.87, \mathrm{p}<0.0001$, but they significantly improved over the study period $\left(t_{37}=2.56, p=0.015\right)$. Conclusion: Yearlong, routine exposure to the LR of involuntarily admitted psychiatric patients may attenuate negative nurse beliefs and attitudes towards $L R$ of such patients at DPB hearings. The more positive attitudes may enhance the nurses' ability to present information about patients without jeopardizing their human and civil rights, and help patients to attain a greater sense of control over their illness management and satisfaction with the services received.
\end{abstract}

Keywords: Attitudes; Changes over Time; Legal Representation; Patient Advocacy; Psychiatric Nurses

\section{INTRODUCTION}

In Israel psychiatric hospitalizations, both voluntary and compulsory, are regulated by the Mental Patients Treatment Act, 1991 [1]. The District Psychiatric Board (DPB) reviews all involuntary commitments, both in the civil and criminal pathway. The DPB is entitled to decide whether extending or not the involuntary hospitalization in the civil pathway and to rule on matters of leave, hospitalization extension or discharge in the criminal pathway [2].

At present there are 3200 hospital beds for psychiatric inpatients in Israel. Approximately 20,000 patients are admitted to psychiatric inpatient care each year. One fourth receives compulsory psychiatric care under either District Psychiatrist Order, or Court Order [3]. DPBs deal with about 8000 cases per year. Patients hospitalized under District Psychiatrist Order appear before the DPB within 14 days after involuntary hospitalization and every three months thereafter. Patients hospitalized under Court Order appear before the DPB every six months or whenever the patients or the staff wish to obtain release, short leave or vacation.

Since 2004, all patients appearing before a DPB are entitled to receive legal representation (LR). Its purpose is to safeguard the rights of the mentally ill facing compulsory commitment and to help them express their opinion on the treatment received and their wishes for discharge from involuntary hospitalization [1].

Psychiatric nurses are challenged not only to provide optimal care but also to protect the human and civil rights of all patients under their care. They are especially challenged to balance the interests of individual patients within the context of the interests of other patients, staff 
members and different stakeholders. What they write or say in their reports on patients' mental state or behavior is commonly used, at least partly, to make legal decisions concerning patients' detainment or discharge. The ethical-legal implication is that nurses are supposed to accurately report patients' mental condition while preserving their civil rights and dignity.

By law since 2004 mental patients in Israel started to receive some LR service whenever appearing before a DPB [4]. This important amendment to the law was the result of a number of factors: 1) the growing criticism on the functioning of the DPBs in the preceding decade by lawyers, judges and psychiatrists; 2) the legislation of the basic constitutional Law on Freedom and Dignity, 3) the recommendations of the "Schnitt Committee", a specially appointed Board whose goal was to assess the functioning of the DPB. All these lead to the proposal that every person appearing before Psychiatric Boards should be legally represented.

Despite its importance, the LR of mentally ill patients committed under a Compulsory Admission Order remains an understudied issue [2]. For example, it has not become clear yet whether the routine practice of LR in psychiatric hospitals has any impact on nurses' knowledge of human rights in general and on their attitudes towards an individual patient civil rights. We are not aware of any study on psychiatric nurse attitudes dealing with LR of psychiatric inpatients before a DPB. Nor are we aware of any study comparing psychiatric nurse attitudes towards LR of psychiatric patients with those of other mental health professionals.

The specific aims of this pilot study were to: 1) compare baseline psychiatric nurses attitudes towards LR of involuntary inpatients before a DPB with those of other mental health professionals, and 2) determine whether the routine implementation of LR of psychiatric inpatients led to any change in the professional groups' attitudes towards the LR of such patients.

\section{BACKGROUND}

\subsection{Attitudes toward Mental Illness and Psychiatric Treatment}

The literature on the attitudes toward medico-legal aspects of psychiatry among various health professional groups is scarce and exclusively descriptive. Nevertheless the literature does point to care-givers attitudes in mental health as focusing on positive outcomes, as well as satisfaction with mental health services. In their literature review, Creech and Pewett [5] noted a positive association between the attitudes of carers towards patients and effective mental health care delivered. The researchers then compared the attitudes of the different mental health professionals (doctors, social workers, rehabilitation staff, nurses, etc.) in psychiatric hospitals and in community mental health centers. Participants attitudes were assessed according to five dimensions: authority and its impact; benevolence; ideology indicating the carer's positive orientation towards mental illness and the mentally ill; restrictiveness (a tendency to perceive patients as a threat to society requiring restriction of their social activity), and belief that mental illness arises from particular interpersonal experiences. Findings from the research highlighted a wide range of beliefs and outlooks held by mental health carers with regard to the causes, treatment and prognosis of mental illness. Interestingly the authors noted that a positive and humane orientation towards mental illness had a positive impact on treatment outcomes. The researchers concluded that positive attitudes toward mental illness and treatment especially among nurses would facilitate better rehabilitation outcomes than authoritative or paternalistic attitudes.

Tay and associates [6] explored the factors influencing nurses' attitudes and involvement with psychiatric patient care. Their aim was to establish which facilitating factors were associated with the most positive nurses' attitudes. The authors found that 31-to-50 year-old nurses with an M. A. diploma, mental health training, and 10 years of psychiatric nursing experience held more positive attitudes towards patients with mental illness that their less educated and experienced counterparts. They also found that senior-level nursing managers had more positive attitudes than staff-level nurses or assistant nurses. Moreover, nurses working in short-stay acute ward environments revealed more positive attitudes than nurses working in long-stay chronic ward environments.

\subsection{Advocacy}

Thomas [7] compared psychiatrists and psychiatric nurses' views of mental health advocacy and found positive support for the perceived usefulness of the approach in both groups. Psychiatrists tended to make negative comments about it, and believed that advocacy had no impact on patient management, but they did not provide any indication that advocates would promote an antimedical-establishment political agenda.

The psychiatrists also indicated that the representation of patients' interests by lawyers would inevitably cause conflicts with the psychiatrists treating them. Nurses, on the other hand, thought that advocacy empowered clients by giving them greater control over the management of their illness. Nurses indicated that advocates challenged their personal opinion about patients' management. The nurses also described positive experiences with advocates who put forward their clients' views, particularly in situations where the client found it difficult to express 
his/her own ideas.

Some nurses, however, cited negative advocacy experiences, for instance, when overzealous advocates urged a client's point of view without actually attempting to mediate as expected by them. By mediation they meant acting as a go-between two parties i.e. the staff member and the patient, in a dispute, in which case advocates should be expected to represent both the professional and the patient viewpoint. Some nurses also reported that they would be failing their professional duty if they represented a patient's view themselves.

\subsection{Legal Representation}

According to Oladimeji [8] the LR of psychiatric patients should not only protect patient rights but also promote patient autonomy and welfare by spelling out wishes, needs, and interests of the patients being represented. In this regard, legal advocates should also collaborate with professional carers in making decisions about a patient treatment and then explain the decision-making process and the decisions made to the patient. Lawyers representing a patient at a DPB hearing typically ground their arguments for or against a patient's detention in hospital, at least partly, on the reports on the his current mental state and behavior of nurses, physicians, and significant others. Healthcare professionals, including nurses, thus need to be well instructed and have thorough knowledge on patients civil and human rights especially of those who must appear at DPB hearings. But are all nurses sufficiently informed or have an appropriate professional attitude toward LR of the human and civil rights of involuntary patients in psychiatric hospital setting? Would LR of patients in the hospital setting result in some nurses feeling threatened or relieved, or fearing for their patients perceived harm or being optimistic about the well-being of their patients? These issues are controversial and need to be further assessed.

\section{METHODS}

\subsection{Study Design}

A case-control pilot survey was designed to determine baseline beliefs and attitudes of hospital mental health professionals towards the LR of involuntarily hospitalized psychiatric patients appearing before a DPB. A longitudinal study design was used to determine possible changes in the participants' attitudes over one year time, following routine implementation of the practice of LR of patients appearing before a DPB at the Tirat Carmel Mental Health Center in Israel. The Institutional Review Board approved the research protocol and written informed consent was received from all participants. The study was initially planned as a pilot study, and after evaluation of the preliminary data a longitudinal study, involving multiple mental health centers across the country was planned.

\subsection{Participants and Setting}

The setting for this study was a state psychiatric hospital which has a total of 228 beds distributed as follows: 24 adolescents' beds, 68 chronic and 136 acute patients' beds, and 14 ambulatory centers. The hospital serves a catchment area with a mixed lower and middle-income population of 600,000 . During the survey period, the professional staff of the hospital included 83 psychiatric registered nurses, 21 certified psychiatrists, 11 psychiatric residents, and 14 social workers. LR was implemented in the hospital since November 1, 2007. Over the 1 -year course of the study, 51 DPB sessions have been conducted, and 50 involuntarily hospitalized patients received routine $L R$.

\subsection{The Questionnaire}

An "Attitudes to Legal Representation Questionnaire" (ALRQ) was especially developed for the study (see Appendix). The questionnaire consists of 11 items grouped into two subscales: the positive and negative attitudes subscales. The first reflects staff member notions about the various benefits bound to legal representation of all participants in the process, whereas the second subscale defines staff members' concerns/beliefs about the potential harm of LR. Correspondingly, the positive attitudes subscale consists of 7 items: "LR helps protect patients' rights"; "LR enables the caring staff to explain care decisions to patients"; "LR makes DPB hearings more careful"; "LR makes the DPB similar to court hearings"; "LR improves the personnel-patient partnership"; "LR improves the quality of psychiatric examinations"; and "LR enables a more accurate evaluation of patients' dangerousness". The negative subscale comprises 4 items: "LR leads to premature discharge from hospital"; "LR increases a likelihood of readmission"; "LR turns DPB hearings into a formal juridical procedure", and "LR distorts the DPBs' decisions".

In the questionnaire, a respondent is asked to assess his/her degree of agreement with each item. The responses are scored on a 4-point Likert scale: 'Completely disagree' (score 1), 'Disagree' (score 2), 'Agree' (score 3), and 'Completely agree' (score 4). Mean scores for each subscale are calculated by summing item scores divided by number of completed items. The cut off point for the operational definition of positive and negative attitude subscale score was established as 2.5. Thus, a mean score lower than 2.5 indicates participant agreement with the subscale items, whereas a mean score of 2.5 and over displays his/her disagreement. The internal consistency of the ALRQ items, as measured by Cron- 
bach's alpha coefficients, was 0.78 for the Positive Attitude subscale and 0.73 for the Negative Attitude subscale.

\subsection{Procedure}

All professional staff members employed at the research site were personally approached by the social worker (TL) who explained the study goals and dispensed the questionnaire among those who gave written informed consent to participate in the study $(n=90)$. All respondents completed the study questionnaire anonymously, 30 days before implementation of the routine practice of legal representation for mental patients (November 1, 2007), and one year later. Respondents remained anonymous, stating only their profession and whether they personally knew any lawyer that represented patients at DPB hearings. Note here, no one reported familiarity with the lawyers.

\subsection{Data Analysis}

Statistical analysis was performed using SPSS, version 15.0 (SPSS Inc, Chicago, IL). Descriptive statistics were used to examine item and subscale scores on the "Attitudes to Legal Representation Questionnaire." One-way analysis of variance (ANOVA) with Tukey HSD post-hoc single comparisons was used to determine statistical significance of baseline differences in the attitude scores of psychiatric nurses and other professionals. Two-tailed, paired t-tests were used to assess changes in the attitude scores of the groups over the study time. An alpha of 0.05 was used to determine statistical significance.

\section{RESULTS}

A total of 67 respondents completed the study questionnaire twice: one month before and one year after the LR was implemented. The participants consisted of 37 psychiatric registered nurses and 30 other mental health professionals (18 psychiatrists and 12 social workers). Response rate was $74 \%$ for the entire sample, $98 \%$ for nurses, and $65 \%$ for other professionals. At baseline, $64 \%$ of all respondents (43 out of 67 ) endorsed the positive attitudes items on the ALRQ and 55\% (37 of 67) endorsed negative attitudes items on the scale.

To test the hypothesis that psychiatric nurses do not differ from other professionals by their attitudes about the LR of hospitalized psychiatric patients at DPB hearings, we compared baseline attitudes' scores between the two groups (Table 1, the baseline column comparison). One way ANOVA, with Tukey post-hoc single comparisons, indicated that initially psychiatric nurses endorsed less positive attitudes $\left[\mathrm{F}_{67,3}=5.28, \mathrm{p}=0.03\right]$ and more negative attitudes $\left[\mathrm{F}_{67,3}=6.87, \mathrm{p}<0.001\right]$ compared to other professionals.

To test the hypothesis that the routine practice of LR of patients before DPB hearings has an effect on nurses' attitudes, we compared their baseline and 1-year follow-up scores (Table 1, line comparisons). Paired t-tests indicated a significant reduction in negative attitudes' scores for the entire sample $\left(\mathrm{t}_{67}=2.51, \mathrm{p}<0.015\right)$. This change was due to a significant decrease in the negative attitudes' scores of the psychiatric nurses $\left(\mathrm{t}_{37}=2.56, \mathrm{p}<\right.$ 0.015). No significant changes were found in scores of the negative and positive attitudes in the comparison group.

Table 1. Initial scores on the attitudes to legal representation questionnaire and their changes over a 1-year study period: Psychiatric nurses vs. other professionals.

\begin{tabular}{|c|c|c|c|c|c|c|}
\hline \multirow{2}{*}{ Hospital staff group } & \multicolumn{2}{|c|}{$\begin{array}{l}\text { Before legal representation } \\
\text { introduced }(\mathrm{N}=67)\end{array}$} & \multicolumn{2}{|c|}{$\begin{array}{c}\text { One year after legal representation } \\
\text { introduced }(\mathrm{N}=67)\end{array}$} & \multicolumn{2}{|c|}{ Paired t-test } \\
\hline & Mean & $\mathrm{SD}$ & Mean & SD & t-value & $\mathrm{p}$ \\
\hline \multicolumn{7}{|l|}{ Psychiatric nurses $(n=37)$} \\
\hline Positive attitudes & 2.44 & 0.38 & 2.35 & 0.47 & 0.87 & 0.38 \\
\hline Negative attitudes & 2.71 & 0.54 & 2.40 & 0.57 & 2.56 & 0.015 \\
\hline \multicolumn{7}{|l|}{ Other professionals $(n=30)$} \\
\hline Positive attitudes & 2.46 & 0.50 & 2.15 & 0.48 & 0.45 & 0.65 \\
\hline Negative attitudes & 3.00 & 0.38 & 3.01 & 0.48 & 0.02 & 0.98 \\
\hline \multicolumn{7}{|l|}{ Total sample $(n=67)$} \\
\hline Positive attitudes & 2.41 & 0.45 & 2.33 & 0.48 & 0.88 & 0.38 \\
\hline Negative attitudes & 2.86 & 0.55 & 2.66 & 0.57 & 2.51 & 0.015 \\
\hline \multicolumn{7}{|c|}{ ANOVA with Tukey post-hoc single comparisons } \\
\hline Positive attitudes & \multicolumn{2}{|c|}{$\mathrm{F}_{3}=6.87, \mathrm{p}<0.001$} & -- & -- & -- & -- \\
\hline Negative attitudes & \multicolumn{2}{|c|}{$\mathrm{F}_{3}=5.28, \mathrm{p}=0.03$} & -- & -- & -- & -- \\
\hline
\end{tabular}




\section{DISCUSSION}

In this study we compared the attitudes of four psychiatric hospital staff groups regarding the LR of patients at DPB hearings before and after its introduction as a routine hospital policy. Our findings indicate that psychiatric nurses held significantly more negative baseline attitudes than other professional groups. The findings indicate that the nurses also demonstrated a significant change in their negative attitudes one year after the implementation of the policy. It thus appears that the implementation of the LR at the hospital and the nurses' everyday exposure to the practice had attenuated the nurses' initial apprehension about the policy one year later.

One explanation for the study findings suggests that routine exposure to the LR of patients at DPB hearings convinced nurses that their initial attitudes were not justified. The innovative introduction of LR may have caused initial anxiety among nurses with a negative attitude, partly due to their ignorance about the impact of this procedure on the ward atmosphere and on their every day practice. Moreover, the contact with lawyers was something new and unknown to them thus arising initial antagonism. Nurses typically have significantly more time for and closer contact with patients than other staff professionals. Their more close day-to-day involvement or more detailed knowledge of the practical needs of patients being represented at DPB hearings may have influenced changes in their attitude towards LR of the patients. Their greater response rate in this pilot study relatively to other professional groups indirectly supports this explanation.

In this pilot study, psychiatric nurses held significantly more negative attitudes about LR of patients than psychiatrists and social workers. It is possible that the nurses were initially more apprehensive than other staff groups regarding the perceived potential of LR to result in harm. Daily exposure to LR and the experience with the practice acquired during one year may all have desensitized the nurses resulting in increased positive attitudes. The attitudes of other professional groups may not have changed because they were already more positive at the beginning or less likely to need enhancement because of ceiling effects.

Noteworthy, nurses' negative attitudes changed over the course of one year while their initial positive attitudes remained unchanged. This finding points to the possibility that negative nurses' attitudes about LR of psychiatric inpatients are not as stable as positive ones, and are therefore amenable to change with exposure to the practice over a relatively short period of time. This finding is inconsistent with Tay et al. [6], who found that enhanced positive nurse attitudes towards caring for people with mental illness was associated with ten or more years of psychiatric nursing experience.

Several limitations of the study should be addressed. The relatively small size of the groups could diminish the statistical power of our analysis. It is possible that pre-post differences would be statistically significant if the sample size of each group had been larger. Another limitation is that no data on background characteristics of the sample were collected because of the anonymity of the survey. Likewise, it was impossible to prevent exposure of participants to other participants within group and between groups, a fact which might have affected the findings. Unfortunately, we were not able to assess the opinions of the lawyers, both members of the DPBs and those who represented mental patients before DPBs, and hence we cannot present their side. This important side of the medicolegal dilemma warrants further investigation.

\section{CONCLUSION AND IMPLICATIONS}

According to the results of our pilot study, routine exposure to the LR of involuntarily admitted psychiatric patients may attenuate negative nurse beliefs and attitudes towards LR at DPBs hearings. Their more positive attitudes may enhance their ability to present adequate information about patients without jeopardizing their human and civil rights.

Our study findings are consistent with qualitative research describing the positive experiences of psychiatric nurses with individuals who advocate on behalf of psychiatric patients [7]. In that research, the nurses stressed the usefulness of advocacy for empowering their clients, giving them greater control over the management of their illness, and stimulating the nurses own thinking about patient management. Thus, it is possible that enhanced positive nurses' attitudes about the LR of mental patients before a DPB may affect their patients' symptom-management and create more satisfaction with the services received.

Further research is needed in order to assess how such attitudes may be influenced and changeable for all professional groups-over a longer time-period. In order to diminish the negative attitudes of the staff a preliminary seminar addressing the initial fears and prejudices towards LR, illustrating the law amendment, its rationale and the procedure of its implementation is recommended. The seminar should also include a discussion group with some lawyers from the Public Defender Office or from the Legal Aid such allowing some desensitization and a better cooperation between the two professions. Illustrations of some case vignettes showing the positive impact of the LR on the patients health should also be recommended. We do believe that in order to better safeguard 
mental patients rights ongoing and periodical meetings should be organized including both hospital staff and representing lawyers, thus promoting improved clinical and legal practice.

\section{ACKNOWLEDGEMENTS}

Dr. A.M. Ponizovsky was supported by the Ministry of Immigrant Absorption. We thank Dr. I. Levav for his support in this study.

\section{REFERENCES}

[1] State of Israel (1991) Mental patients treatment act. Jerusalem.

[2] Bauer, A., Rosca, P., Grinshpoon, A., Khawalled, R. and Mester, R. (2005) District psychiatric boards in Israel: Expectations and realities. The Journal of Forensic Psychiatry, 28, 661-669.

[3] Ministry of Health. (2009) Mental health in Israel, Statistical Annual 2008. Ministry of Health, Jerusalem.

[4] Bauer, A., Khawaled, R., Rosca, P. and Ponizovsky, A.
(2008) Legal representation is associated with psychiatric readmissions. Open Law Journal, 1, 6-10.

doi:10.2174/1874950X00801010006

[5] Creech, S.K. and Prewett, R.C. (1976) Opinions about mental illness held by personnel of a state psychiatric hospital and community mental health centers. Journal of Community Psychology, 4, 347-356.

doi:10.1002/1520-6629(197610)4:4<347::AID-JCOP229 0040405>3.0.CO;2-E

[6] Tay, C., Pariyasami, D., Ravindran, K., Ali, M. and Rowsudeen, M. (2004) Nurses attitudes to people with mental illness in a psychiatric hospital in Singapore. Journal of Psychosocial Nursing and Mental Health Services, 42, 40-47.

[7] Thomas, Ph. (2001) A survey of psychiatrists and nurses views of mental health advocacy. Psychiatric Bulletin, 25, 477-448.

[8] Oladimeji, K. (1999) The value of advocacy: Putting ethics into practice. Psychiatric Bulletin, 23, 569-571.

\section{APPENDIX}

\section{The Attitudes to Legal Representation Questionnaire}

The new Article 29(a) of the Mental Patients Treatment Act, 1991, requires to provide legal representation of involuntarily hospitalized mentally ill persons before a District Psychiatric Board. In order to understand the attitude of mental health professions to legal representation, we ask you to note to which extent you agree or disagree with the following statements.

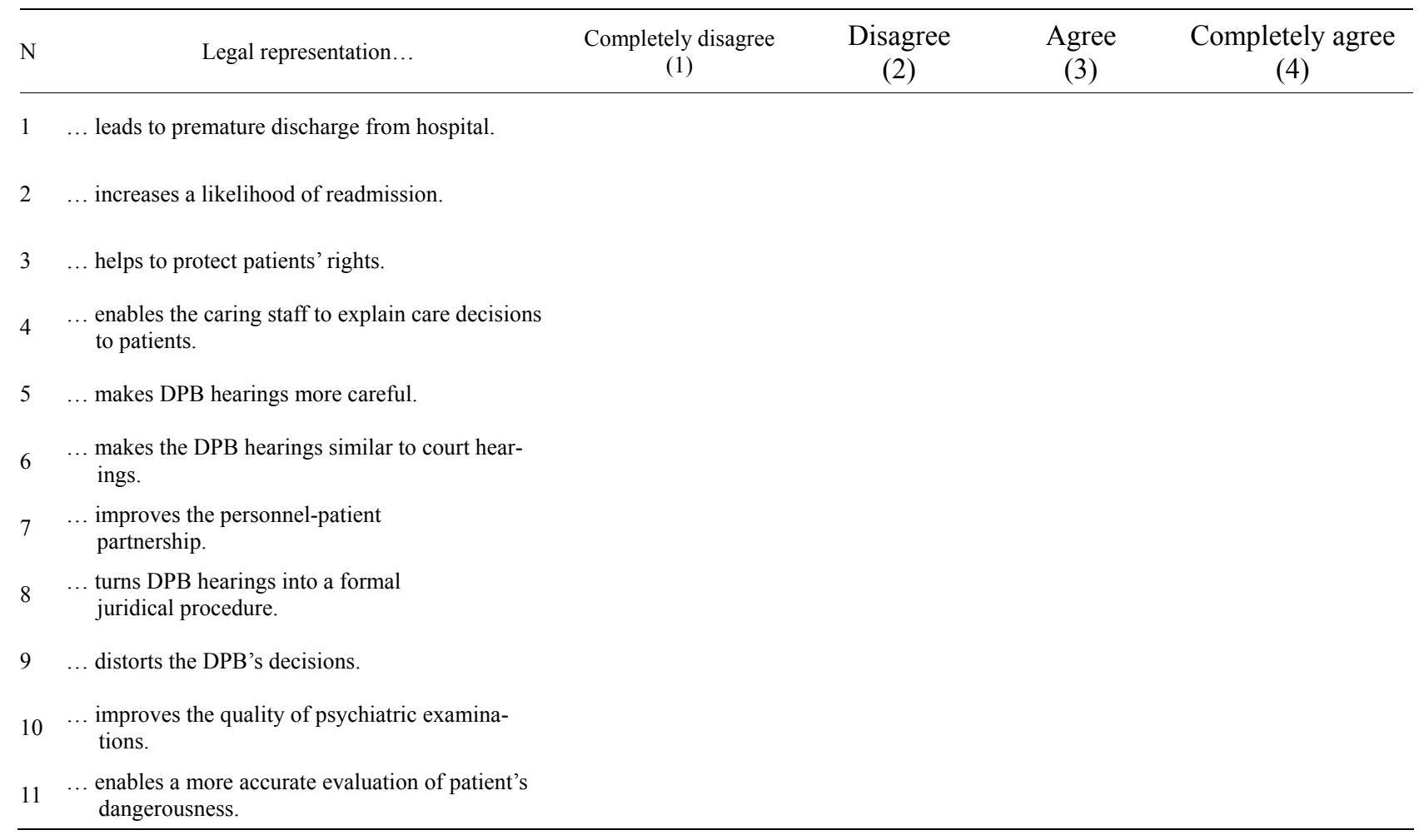

Scoring: The Negative attitudes index is the sum of scores for items 1,2, 8, and 9; The Positive attitudes index is the sum of scores for items 3, 4, 5, 6, 7, 10 and 11 . 